\title{
Schuldhafte Herbeiführung des Versicherungsfalles bei Vorliegen des ausgeprägten obstruktiven Schlafapnoe-Syndroms nach dem neuen VVG?
}

\author{
Does the Sleep Apnoea Sufferer Act Grossly Negligent According to Paragraph 81 of the New German \\ Insurance Contract Act (VVG) when he Causes a Traffic Accident through Microsleep?
}

Autor

Institut

\section{E. Fromm}

Rechtsanwalt und Fachanwalt für Strafrecht, c/o Kanzlei Dr. Caspers \& Mock eingereicht 1.12 .2008

akzeptiert nach Revision

2.1.2009

Bibliografie

DOI 10.1055/s-0028-1119573

Online-Publikation: 3. 3. 2009

Pneumologie 2009; 63:

222-227 @ Georg Thieme

Verlag KG Stuttgart · New York ISSN 0934-8387

Korrespondenzadresse Dr. jur. Ingo E. Fromm

Rechtsanwalt und Fachanwalt für Strafrecht

c/o Kanzlei Dr. Caspers \& Mock Rudolf-Virchow-Str. 11

56073 Koblenz

fromm@caspers-mock.de

\section{Zusammenfassung \\ $\nabla$}

Das ausgeprägte obstruktive Schlafapnoe-Syndrom kann zu schwersten Verkehrsunfällen führen, wenn der Fahrer am Steuer seines Kfz einschläft. Der Beitrag befasst sich mit den versicherungsrechtlichen Folgen, insbesondere mit der Problematik, ob dem Versicherungsnehmer der Einwand der groben Fahrlässigkeit seitens des Versicherers droht. Seit dem 01.01.2008 gilt das neue Versicherungsvertragsgesetz, welches in $\S 81$ bestimmt, dass die Leistung bei grob fahrlässiger Herbeiführung des Schadensfalles entsprechend dem Grad des Verschuldens des Versicherungsnehmers prozentual zu kürzen ist. In der Literatur wird vorgeschlagen, innerhalb dieses Verschuldensgrades abzustufen in ,geringe“, „mittlere“ oder „gravierende“ grobe Fahrlässigkeit. In der Regel mag ein Kraftfahrer, bevor er am Steuer seines Fahrzeugs während der Fahrt einschläft, nach dem gegenwärtigen Stand der ärztlichen Wissenschaft deutliche Zeichen der Ermüdung an sich wahrnehmen. Das Missachten solcher Anzeichen indiziert grundsätzlich das Vorliegen grober Fahrlässigkeit. Hat der Fahrer derartige offensichtliche Ermüdungserscheinungen pflichtwidrig ignoriert, so dürfte der Versicherer seine Leistung prozentual kürzen. Nickt ein Kraftfahrzeugführer nach langer Autofahrt trotz festgestellter Müdigkeit hinter dem Steuer ein, so stellt dies eine mittlere grobe Fahrlässigkeit dar, sodass die Leistung um mind. 50\% zu kürzen ist. Handelt nun der Schlafapnoiker aber stets grob fahrlässig bei einem krankheitsbedingten „Sekundenschlaf"? Diese Fragestellung ist in der Rechtsprechung nicht abschließend zugunsten des Versicherungsnehmers entschieden worden. Die obergerichtliche Rechtsprechung erkennt zwar, dass bei einer krankhaften (Schlaf-)Störung offensichtliche Ermüdungserscheinungen nicht zwangsläufig vorausgehen müssen. Nach der Auffassung des Verfassers muss hier aber genauer zwischen behan-

\section{Abstract}

The strongly pronounced obstructive sleep apnoea syndrome can lead to serious traffic accidents if the driver falls asleep at the wheel. This article deals with the insurance law consequences, especially with the problem if the insurer raises the objection that the sleep apnoea sufferer acted grossly negligent. Since the new German Insurance Contract Act of $1^{\text {st }}$ January 2008 paragraph 81 regulates a benefit reduction in cases of gross negligence by the insurant. The insurer can shorten assurance benefit according to the degree of fault. In the literature it is proposed to grade the level of grossly negligence into low, middle and serious forms of default. Usually the driver notices clear signs of exhaustion in the current state of medical science. If he ignores obvious facts of exhaustion the insurer can shorten assurance benefits percentual. When the driver dozes off after a long trip although he has noticed his exhaustion, a case of gross negligence exists. Here the insurer can reduce the assurance benefit by about $50 \%$. Does the sleep apnoea sufferer always act in a grossly negligent manner in cases of microsleep? This point has not yet been decided by the German jurisdiction in favour of the policyholder. The jurisdiction is aware of the fact that in the case of sleep apnoea fatigue does not necessarily need to precede the act of falling asleep. In the opinion of the author it has to be distinguished between medicated and not medicated sleep apnoea and further if the sufferer knows about his disease. If the sleep apnoea sufferer has known of the risk of sudden microsleep, for example, after being warned by his doctor who discovered the disease, gross negligence cannot be dismissed and assurance benefit has to be reduced by about $65 \%$. 
delter und unbehandelter Schlafapnoe sowie der Frage, ob Kenntnis vom Krankheitsbild besteht, differenziert werden. Hat der Schlafapnoiker etwa einen Unfall durch Einnicken am Steuer verursacht und war ihm das Risiko des plötzlichen Auftretens von Schläfrigkeit bewusst, etwa weil ihn sein Arzt über die Gefahr aufgeklärt hat, so kann eine gravierende grobe Fahrlässigkeit nicht von der Hand gewiesen werden mit der Folge einer Kürzung um bis zu $65 \%$.

\section{Einführung in die Problematik}

Beim obstruktiven Schlafapnoe-Syndrom handelt es sich bekanntlich um nächtliche Atemregulationsstörungen durch einen Verschluss der oberen Atemwege (Pharynxkollaps), an dem im mittleren Alter $4 \%$ aller Männer und $2 \%$ aller Frauen leiden. Es handelt sich um eine chronische Erkrankung, die sich in den meisten Fällen nicht mehr zurückbildet. Die wichtigsten Symptome sind neben erhöhter Tagesmüdigkeit und zwanghaften Einschlafattacken das laute nächtliche Schnarchen.

Führt der Schlafapnoiker unter dem Einfluss von gestörtem Schlaf und exzessiver Tagesmüdigkeit einen Versicherungsfall herbei, so ist zwischen Versicherungsnehmer und Versicherer oft umstritten, ob der Unfallverursacher den eigenen Fahrzeugschaden von seiner Kaskoversicherung ersetzt bekommt. Regelmäßig bedarf dieser Streitpunkt der gerichtlichen Klärung. Das Einschlafen am Steuer eines Kfz gehört nämlich objektiv zu den schwersten Verkehrsverstößen. Die Frage der Schadensersatzleistung ist umso wichtiger, als solche Unfälle oft zu Totalschäden am eigenen Auto führen. Im Versicherungsrecht droht ihm seitens der Kfz-Kaskoversicherung der Einwand der groben Fahrlässigkeit. Rechtliche Grundlage hierfür war bis zum 01.01.2008 $\S 61$ Versicherungsvertragsgesetz (VVG) a.F. ${ }^{1}$, der bei vorsätzlicher oder grob fahrlässiger Herbeiführung des Schadensfalles vorsah, dass der Versicherer von seiner Leistungspflicht ganz frei wurde. Es bestand eine strikte Grenze zwischen einfach fahrlässigem und grob fahrlässigem Verhalten des Versicherungsnehmers. Während bei einfacher Fahrlässigkeit die volle Versicherungsdeckung bestehen blieb, wurde der Versicherer schon bei grober Fahrlässigkeit vollständig von der Leistung frei. Weil die Trennung zwischen einfacher und grober Fahrlässigkeit im konkreten Einzelfall selten so eindeutig ist wie die daran geknüpfte Rechtsfolge, war das sog. „Alles-oder-Nichts-Prinzip“ Gegenstand ausufernder konkretisierender und auch korrigierender Rechtsprechung. Das starre „Null oder Hundert“-System wurde als ungerecht empfunden. Teilweise wurde die Rechtslage als gegen den Verhältnismäßigkeitsgrundsatz verstoßend und damit verfassungswidrig angesehen [1].

Nach dem neuen Versicherungsvertragsgesetz gibt es das „Allesoder-Nichts-Prinzip“ nicht mehr, der Versicherer darf nach $§ 81$ Abs. 2 VVG n.F. seine Leistung bei grober Fahrlässigkeit nur noch entsprechend dem Grad des Verschuldens des Versicherungsnehmers kürzen. Die Neuregelung soll im Einzelfall ermöglichen, den jeweiligen Schutzinteressen des Versicherungsnehmers Rechnung zu tragen.

Wie praktisch relevant dieser Problemkreis für die Versichertengemeinschaft ist, lässt sich allein daran ablesen, dass laut Unfallstatistik im Jahr 2005 insgesamt 1700 Unfälle auf Sekundenschlaf

\footnotetext{
1 §61 VVG alte Fassung (a.F.) lautete: „Der Versicherer ist von der Verpflichtung zur Leistung frei, wenn der Versicherungsnehmer den Versicherungsfall vorsätzlich oder durch grobe Fahrlässigkeit herbeiführt.“
}

zurückzuführen waren und nach Angaben der Bundesanstalt für Straßenwesen ${ }^{2}$ Übermüdung die zweithäufigste Ursache für Lkw-Unfälle ist. Schlafapnoiker haben eine siebenmal höhere Unfallrate im Vergleich zu anderen Verkehrsteilnehmern, womit dieses Krankheitsbild als hohes Risiko für Autounfälle identifiziert ist [2].

Der Verfasser setzt sich mit der äußerst komplexen und vielschichtigen Rechtslage bei Unfällen durch Einnicken am Steuer auseinander und geht konkret auf den - durch eine Schlafapnoe verursachten - „Sekundenschlaf“ ein und nimmt Stellung zu den umstrittenen Fragen der Leistungskürzung bei grober Fahrlässigkeit, der Beweislastverteilung sowie der Streitfrage, ob der Schlafapnoiker überhaupt schuldhaft handelt und unter welchen Voraussetzungen eine verminderte Schuldfähigkeit zum Wegfall der groben Fahrlässigkeit führen kann. Den Darstellungsschwerpunkt bildet die Frage, welche quotalen Kürzungen nach Einführung des neuen Versicherungsvertragsgesetzes beim Einnicken am Steuer infolge des Schlafapnoesyndroms vorzunehmen sind.

\section{Definition der groben Fahrlässigkeit nach VVG $\nabla$}

Grobe Fahrlässigkeit liegt nach allgemein anerkannter Rechtsprechung in der Regel dann vor, wenn die im Verkehr erforderliche Sorgfalt in besonders schwerem Maße verletzt wird und wer das unbeachtet lässt, was im gegebenen Fall jedem hätte einleuchten müssen. ${ }^{3}$ Sowohl in objektiver Hinsicht muss das gewöhnliche Maß an Fahrlässigkeit erheblich überschritten werden als auch in subjektiver Hinsicht ein unentschuldbares Fehlverhalten vorliegen. Die Annahme grober Fahrlässigkeit auf der subjektiven Seite setzt voraus, dass die im Verkehr erforderliche Sorgfalt durch ein auch subjektiv unentschuldbares Verhalten in hohem Maße außer Acht gelassen worden ist, weshalb ein in subjektiver Hinsicht gesteigertes Fehlverhalten aufgrund der Würdigung aller Tatumstände festgestellt werden muss [3]. ${ }^{4}$ Die Handlungsweise des Versicherungsnehmers muss sich auf die Herbeiführung des Versicherungsfalls kausal ausgewirkt haben [1]. Die grobe Fahrlässigkeit ist vom Versicherer zu beweisen. Dabei bezieht sich die Beweislast sowohl auf die objektiven als auch die subjektiven Voraussetzungen. ${ }^{5}$

\section{Die Rechtslage nach dem neuen Versicherungs- vertragsgesetz \\ $\nabla$}

Das neue VVG ist am 01.01.2008 in Kraft getreten. ${ }^{6}$ Es ist auf alle Versicherungsverträge anwendbar, die ab 01.01.2008 geschlossen wurden. Die alte Version des VVG behält weiterhin seine Bedeutung, da es für die bis zu diesem Zeitpunkt bereits bestehenden Versicherungsverträge bis zur Übergangszeit von einem Jahr, also bis 31.12.2008 anzuwenden ist [4]. Nach dem neuen Versicherungsvertragsgesetz ist der Versicherer gem. §81 Abs. 1 weiterhin unverändert nicht zur Leistung verpflichtet, wenn der Versicherungsnehmer vorsätzlich den Versicherungsfall herbeiführt.

\footnotetext{
2 Bericht M 174.

3 Zuletzt BGH VersR 1992, 1087 = RuS 92, 279; VersR 1989, 582; ständige Rechtsprechung des BGH seit BGHZ 10, 14; vgl. etwa auch BGH VersR 1986, 671; 1988, 509; 1989, 141.

${ }^{4}$ BGH, NJW 1985, 2648 = NVersZ 1985, 440 m.w. Nachw.

${ }^{5}$ Ganz h.M.: BGH, NJW 1989, 1612 = VersR 1989, 469; NJW 1990, 2387 . BGHZ 65, 118, 121 f.; BGH NJW 1985, 919, 920; BGH NJW 1985, 917, 918. ${ }^{6}$ Art. 1 EGVVG.
} 


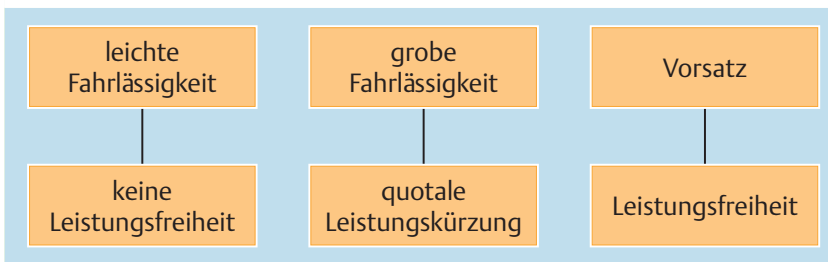

Abb. 1 Darstellung zu §81 VVG.

\section{einfache}

mittlere

gravierende

Abb. 2 Darstellung zur „Kürzung bei grober Fahrlässigkeit“.

Führt der Versicherungsnehmer den Versicherungsfall grob fahrlässig herbei, ist der Versicherer gem. Abs. 2 der Vorschrift befugt, seine Leistung in einem der Schwere des Verschuldens des Versicherungsnehmers entsprechenden Verhältnis prozentual zu kürzen, vgl. Abb. 1. Umstritten ist darüber hinaus, ob und unter welchen Voraussetzungen sogar eine Kürzung „auf Null“ zulässig ist [5].

Zunächst ist die Höhe der zu erbringenden Leistung des Versicherers zu ermitteln, die dann entsprechend der Quote zu reduzieren ist [6]. Wesentliche Bedeutung erlangt damit die Frage, welche Quoten bei grober Fahrlässigkeit zu bilden sind. Es ist hier zu fragen, wie schwer die grobe Fahrlässigkeit im konkreten Einzelfall wiegt und wie viel Leistungsfreiheit die grobe Fahrlässigkeit begründet. Die Praxis steht vor der Problematik, mit dieser Vorschrift umzugehen. Es wird demnächst nur von den Instanzgerichten Urteile geben, wie man es bei Verteilung von Haftungsquoten bei Verkehrsunfällen kennt. Es wird voraussichtlich lange dauern, bis hier Rechtssicherheit herrschen wird. Enormes Streitpotenzial wird es künftig bei der Ermittlung der „richtigen Quote“ in Fällen grober Fahrlässigkeit geben.

Nach der bisher erschienenen Literatur zu $§ 81$ II VVG n.F. muss von nun an eine Gewichtung innerhalb der groben Fahrlässigkeit vorgenommen werden, vgl. $\bullet$ Abb. 2. Innerhalb dieses Verschuldensgrades sei darauf abzustellen, ob das Verhalten des Versicherungsnehmers eher als „einfach“, „mittelgradig“ oder „besonders gravierend“ grob fahrlässig zu werten ist [1]: Liegt das Verschulden eher in der Nähe von leichter Fahrlässigkeit oder Vorsatz? In der Literatur werden Kürzungsquoten von 25\%, 50\% und 75\% diskutiert. Diese Grobaufspaltung ist nach dem Gesetz jedoch fraglich. Die Leistungskürzung knüpft vielmehr an die Schwere des individuellen Verschuldens des Versicherungsnehmers im Einzelfall an. Das kann durch feste Quoten für die Mehrzahl der Fälle nicht hinreichend erfasst werden [7]. Die deutsche Literatur orientiert sich noch - mangels Vorliegens einer eigenen Rechtsprechung - an den Kürzungsquoten des Schweizerischen Bundesgesetzes über den Versicherungsvertrag, das eine Abstufung der Versicherungsleistung nach dem jeweiligen Grad der groben
Fahrlässigkeit schon seit Jahrzehnten vorsieht. ${ }^{7}$ In jedem Falle muss die Schwere des Verschuldens seitens des Versicherers bewiesen werden [6].

\section{Stets grobe Fahrlässigkeit bei einem durch eine Schlafapnoe verursachten „Sekundenschlaf"? \\ $\nabla$}

Nach herrschender Rechtsprechung ${ }^{8}$ liegt im Falle der Verursachung eines Verkehrsunfalls durch bloßes kurzfristiges Einnicken am Steuer (sog. „Sekundenschlaf“) als solches generell noch keine grobe Fahrlässigkeit, sondern „einfache“ Fahrlässigkeit vor. Die Feststellung der groben Fahrlässigkeit erfordert in derartigen Fällen die Überzeugung davon, dass sich der Fahrer über von ihm erkannten deutlichen Vorzeichen des Einnickens, sog. ProdormalErscheinungen, oder über diesbezügliche Bedenken, die sich ihm aufdrängen mussten, hinweggesetzt hat. ${ }^{9}$ Für den Fall der „Übermüdung“ eines ansonsten gesunden Fahrers wird als Kürzungsquote nach dem neuen VVG pauschal 75\% vorgeschlagen (Autobild, Heft vom 29.02.2008).

Umstritten ist in der Rechtsprechung allerdings, ob das Vorliegen solcher Vorzeichen in jedem Einzelfall konkret bewiesen werden muss oder ob es einen allgemeinen Erfahrungssatz dahingehend gibt, dass einem „Sekundenschlaf“ immer Warnzeichen vorausgehen, sodass der Beweis über diesen (allgemeinen) Erfahrungssatz geführt werden kann, und wen die Beweislast hinsichtlich des Vorhandenseins dieser Vorzeichen trifft. ${ }^{10}$

a) Einerseits, so argumentiert die Rechtsprechung, bestehe nach dem gegenwärtigen Stand der ärztlichen Wissenschaft der Erfahrungssatz, dass ein Kraftfahrer, bevor er am Steuer seines Fahrzeugs während der Fahrt einschläft (einnickt), stets deutliche Zeichen der Ermüdung (Übermüdung) an sich wahrnimmt oder wenigstens wahrnehmen kann. Ein Sekundenschlaf ohne Vorzeichen sei so untypisch, dass der Versicherungsnehmer diesen Ausnahmefall beweisen müsse. ${ }^{11}$ Nur besondere Anhaltspunkte, die das Verhalten des Fahrers in einem milderen Licht erscheinen lassen, könnten den Schluss auf grobe Fahrlässigkeit verhindern. ${ }^{12}$ Der Versicherer würde in diesem Fall den Rechtsstreit schon mit der schlichten Behauptung, ein Einschlafen am Steuer finde niemals ohne Vorankündigung statt, gewinnen.

b) Nach der in der verbraucherfreundlicheren obergerichtlichen Rechtsprechung vertretenen Ansicht gehen einem Einnicken am Steuer eines Kfz nicht zwangsläufig unübersehbare Anzeichen voraus, deren Nichtbeachtung in der Regel grob fahr-

\footnotetext{
7 Art. 14 Abs. 2.

8 OLG Koblenz, Urteil vom 11.1.2007 - 10 U 949/06, r +s 2007, 151; BGH NJW 1974, 948; Das OLG Celle ( $\mathrm{r}+\mathrm{s} 2005$, 456) entschied, dass kein Leistungsausschluss wegen grob fahrlässiger Herbeiführung des Versicherungsfalls vorliege, wenn nicht auszuschließen und nach den Umständen darüber hinaus auch naheliegend ist, dass ein Sekundenschlaf ursächlich war, den der Versicherungsnehmer „einfach fahrlässig“ nicht vorhergesehen hat (Fahrt nach 11- statt sonst 9-stündiger Nachtschicht; kein Gefühl der Fahruntauglichkeit; regelmäßige, problemlose Autofahrt nach den Nachtschichten); vgl. auch OLG Düsseldorf, Urteil vom 26.10.2001 - 1 U 73/01, LG Kaiserslautern: Urteil vom 30.06.2004 - 3 O 1176/03.

${ }_{9}^{9}$ BGH VersR 1977, 619, 620; OLG Schleswig DAR 2001 463; OLG Koblenz r+s 1998, 187 f.; OLG Zweibrücken NZV 1998, 289; OLG Oldenburg NJW-RR 1999, 469.

${ }^{10}$ Vgl. zu einem Sonderfall OLG Frankfurt/Main r + s 1993, 290; für Versicherungsnehmer OLG Hamm VersR 1997, 961 f.; auch OLG Saarbrücken NJWRR 2003, $605 \mathrm{f}$.

11 BGH NJW 1970, 520.

12 OLG Hamm, VersR 1997, 961; ZfSch 1998, 182.
} 
lässig sei. Es müssten stets Umstände festgestellt werden können, die den Schluss darauf zulassen, dass der Fahrer sich über von ihm erkannte deutliche Vorzeichen der Ermüdung bewusst hinweggesetzt hat. Nur dann könne das Verschulden des Fahrers als grob fahrlässig eingeordnet werden. ${ }^{13}$

Oft ist zwischen den Parteien des Rechtsstreits bereits strittig, ob der Anspruchsteller überhaupt am Steuer eingeschlafen ist. Von diesem Umstand muss sich das Gericht gem. §286 ZPO überzeugen. Im Streitfall wird es vor dem Zivilgericht auf das Ergebnis eines Sachverständigengutachtens ankommen. Der Gutachter äußert sich - wie in dem vom BGH im Urteil vom 29.10.2003 - IV ZR 16/03 - entschiedenen Fall, in dem es zu einer Kollision infolge eines Rotlichtverstoßes kam - dazu, ob der Anspruchsteller vor dem von ihm verursachten Verkehrsunfall Anzeichen einer Tagesschläfrigkeit bemerkt hat. ${ }^{14}$ Die Klägerin hatte behauptet, ihr Geschäftsführer sei zurzeit des Unfalls schuldunfähig gewesen. Er habe seinerzeit unter einer vor dem Unfall noch unerkannten Atemstörung während des Schlafes gelitten, in deren Folge er bei der Unglücksfahrt kurzzeitig das Bewusstsein verloren habe.

\section{Versicherungsschutz bei behandelter/unbehandelter Schlafapnoe}

Selbst die verbraucherunfreundliche Rechtsprechung billigt dem obstruktiven Schlaf-Apnoiker zu, dass bei einer krankhaften (Schlaf-) Störung offensichtliche Ermüdungserscheinungen nicht zwangsläufig vorausgehen müssten. Die Krankheit besteht schließlich gerade in dem unvorhersehbaren Einschlafen am Steuer. Das OLG Hamm ${ }^{15}$ entschied bereits im Jahr 1953, dass bei einem Unfall durch Einschlafen die Möglichkeit eines unvorhersehbaren Einschlafens anerkannt sei, jedoch zu prüfen sei, ob dem Unfall ein - dem Einschlafenden erkennbarer - Zustand der Ermüdung vorausgegangen sei. Das Missachten solcher Anzeichen indiziere grundsätzlich das Vorliegen grober Fahrlässigkeit. Hiervon ausgenommen seien allerdings Krankheitszustände. Diese Rechtsprechung festigte das BGH in einer Grundsatzentscheidung, in der er feststellte, dass dem Einschlafen, außer bei einer krankhaften Störung, offensichtliche Ermüdungserscheinungen vorausgehen. ${ }^{16}$ Diese Rechtsprechung hat der Bundesgerichtshof in seinem Urteil vom 29.10.2003 fortgesetzt, wobei er entschied, dass der Betroffene die ihm unbekannte Schlafapnoe beweisen müsse. ${ }^{17}$

Im Einzelfall muss jedoch zwischen behandelter und unbehandelter Schlafapnoe näher differenziert werden und nochmals geprüft werden, welches Verhalten des Versicherungsnehmers nicht mehr tolerierbar ist und demzufolge mit der Versagung bzw. quotalen Kürzung der an sich ausbedungenen Versicherungsleistung zu rechnen ist. Bei unbekannter und in dieser Folge unbehandelter Schlafapnoe wird das Verschulden am Sekundenschlaf im Allgemeinen als gering und allenfalls leicht fahrlässig anzusetzen sein, wenn es Vorwarnzeichen krankheitsbedingt nicht gab/geben konnte. In der Tat bleibt die Erkrankung oft jahrelang unentdeckt, so dass der Einwand der Nichterkennbarkeit

\footnotetext{
13 OLG Frankfurt a. M., MDR 1998, 215; OLG Oldenburg, NJW-RR 1999, 469.

${ }^{14}$ NJW-RR 2004, 173.

${ }^{15}$ NJW 1953, 1077.

${ }^{16}$ BGH , Urteil vom 18.11.1969 - 4 STR 66/69, NJW 1970, 520. In dem zugrundeliegenden Fall litt der Betroffene an der seltenen Krankheit der Narkolepsie.

${ }^{17}$ NJW-RR 2004, 173.
}

\section{Tab. 1 Fallbeispiele}

\begin{tabular}{|c|c|}
\hline Sekundenschlaf verursacht durch & Leistungskürzung \\
\hline $\begin{array}{l}\text {... unbekannte unbehandelte Schlaf- } \\
\text { apnoe, krankheitsbedingt keine Vor- } \\
\text { warnzeichen }\end{array}$ & keine \\
\hline $\begin{array}{l}\text {... unerwartet auftretender Fall der } \\
\text { Tagesschläfrigkeit trotz Einleitung von } \\
\text { Behandlungsmaßnahmen (wie CPAP- } \\
\text { Therapie), unerwarteter Rückfall }\end{array}$ & keine (allenfalls fahrlässig) \\
\hline $\begin{array}{l}\text {... Tagesschläfrigkeit trotz Einleitung } \\
\text { von Behandlungsmaßnahmen (wie } \\
\text { CPAP-Therapie), bereits in Vergangen- } \\
\text { heit Tagesschläfrigkeit am Steuer auf- } \\
\text { getreten }\end{array}$ & $\begin{array}{l}\text { bis zu } 35 \% \text { (gering grob } \\
\text { fahrlässig) }\end{array}$ \\
\hline $\begin{array}{l}\text {... lange Autofahrt trotz festgestellter } \\
\text { Müdigkeit ohne Krankheitsbild }\end{array}$ & $\begin{array}{l}\text { ca. } 50 \% \text { (mittel grob } \\
\text { fahrlässig) }\end{array}$ \\
\hline $\begin{array}{l}\text {... typische Anzeichen von Bewusst- } \\
\text { losigkeit, Therapie abgelehnt gegen } \\
\text { ärztlichen Rat }\end{array}$ & $\begin{array}{l}\text { mind. } 65 \% \text { (gravierend } \\
\text { grob fahrlässig) }\end{array}$ \\
\hline $\begin{array}{l}\text {... messbar auffällige Tagesschläfrigkeit, } \\
\text { Fahrerlaubnisbehörde hat Führerschein- } \\
\text { entziehungsverfahren eingeleitet }\end{array}$ & $\begin{array}{l}\text { ggf. Leistungskürzung } \\
\text { auf Null }\end{array}$ \\
\hline
\end{tabular}

nicht unbedingt abwegig ist. Das Schlafapnoesyndrom ist anerkanntermaßen als besonders risikoreich für den Straßenverkehr aufgrund der unfreiwilligen Einschlafneigung einzuschätzen. Der sich bei den Betroffenen über lange Zeiträume täglich wiederholende partielle Schlafentzug bewirkt, dass sie bei fast jeder Gelegenheit schnell einschlafen können. Hat der Betroffene also vor dem von ihm verursachten Verkehrsunfall keine Anzeichen einer Schlafapnoeerkrankung bemerkt, so darf keine Leistungskürzung vorgenommen werden. Dieses Ergebnis wird insbesondere durch die Vorschrift des $\S 827$ II S. 2 BGB gestützt, wonach die zivilrechtliche Verantwortlichkeit und damit eine Verpflichtung zur Haftung nicht eintreten, wenn der Betroffene ohne Verschulden in den Zustand (des Sekundenschlafs) geraten ist.

Das plötzliche Eintreten eines Sekundenschlafs nach einer zuvor völlig symptomlos wachen und leistungsfähigen Fahrt ist nach den Erkenntnissen der Schlafmedizin jedoch eher ungewöhnlich. Plötzlich und unerwartet auftretende Fälle der Tagesschläfrigkeit sind atypisch. ${ }^{18}$ Um ca. 50\% ist die Leistung zu kürzen, wenn typische Anzeichen von Bewusstlosigkeit zum maßgeblichen Zeitpunkt nicht fehlten, vgl. $\bullet$ Tab. 1 mit weiteren Fallbeispielen. Im Allgemeinen wird der Betroffene jedenfalls die als typischen Bestandteil des Syndroms bezeichnete Tagesschläfrigkeit wahrgenommen haben. Hier hätte sich der Fahrer über die Umstände, die die Gefahr des Einnickens erkennbar machen, in besonders vorwerfbarer Weise hinweggesetzt. ${ }^{19}$ Hat er zudem trotz eines auffälligen Befundes eine Therapie gegen ärztlichen Rat abgelehnt, müsste die Rechtsprechung entscheiden, dass sich der Schlafapnoiker sehenden Auges trotz unzureichender Behandlung - also trotz offenkundiger Kenntnis des bestehenden Risikos - in die Gefahr begibt. Dem Versicherungsnehmer wäre in diesem Zusammenhang ein Unterlassen vorzuwerfen, welches nach der Rechtsprechung zu einem Verschulden unter der Voraussetzung führt, dass er „mögliche, geeignete und zumutbare Maßnahmen“ nicht ergreift. ${ }^{20}$ Als zumutbare Maßnahmen wäre hier

\footnotetext{
18 OLG Saarbrücken, r+s 2003, 101, 104.

${ }^{19}$ Hierzu: OLG Oldenburg NVersZ 1999, 80 m.w.N. OLG Frankfurt NZV 1993, 32.

20 BGH VersR 1984, 25; OLG Nürnberg NZV 1990, 315; OLG Oldenburg RuS 1994, 246 = VersR 1994, 1336.
} 
an die Durchführung der Behandlungsform einer CPAP21-Therapie zu denken. Diese bietet zwar keine Heilungsmöglichkeit, sie dient jedoch zur Behandlung vorübergehender Atemstillstände während des Schlafens und führt nach wissenschaftlichen Erkenntnissen im Regelfall bereits nach drei bis vier Wochen zur Wiederherstellung der Fahrtauglichkeit. Der Versicherungsnehmer muss mit einer Leistungskürzung um mindestens $65 \%$ rechnen, da sein Verhalten als besonders grob fahrlässig gewertet werden muss.

Diese Argumentation wird gestützt durch Anlage 4, Ziff. 11.2.1, der Fahrerlaubnisverordnung (FeV), nach der der Betroffene bei messbar auffälliger Tagesschläfrigkeit ungeeignet zum Führen von Kraftfahrzeugen ist und mit dem Entzug der Fahrerlaubnis rechnen muss [8]. Eine Teilnahme am Straßenverkehr müsste als Außerachtlassung der im Verkehr erforderlichen Sorgfalt in besonders schwerem Maße und damit grob fahrlässig beschrieben werden.

\section{Sekundenschlaf als Bewusstseinsstörung gem. §827 S. 1 BGB? \\ $\nabla$}

Grundsätzlich setzt im Zivilrecht jedes haftungsrelevante Verschulden persönliche Verantwortungsfähigkeit (Schuldfähigkeit) voraus. Kann der Schlafapnoekranke für den Schaden überhaupt verantwortlich gemacht werden? Nach der Vorschrift des $§ 827$ S. 1 BGB ist schuldunfähig, wer einem anderen im Zustand der Bewusstlosigkeit einen Schaden zufügt. Besonders im Falle des Sekundenschlafs ohne jede Vorankündigung (Erscheinungen starker Übermüdung oder sonstige bemerkbare Beeinträchtigungen des Leistungsvermögens) kann der Apnoiker bei dem Unfall bewusstlos und damit schuldlos gewesen sein. Wer für diesen Umstand die Beweislast trägt, ist bereits stark umstritten.

a) In der Rechtslehre wird vertreten, dass $§ 827$ S. 1 BGB - mit der in ihm nach allgemeiner Rechtsauffassung ${ }^{22}$ enthaltenen Beweislastregelung, die dem Schädiger die Beweislast für den Ausschluss seiner Verantwortlichkeit auferlegt - bei Anwendung des Risikoausschlusses des $§ 61$ VVG bzw. der quotalen Kürzung des §81 VVG nicht gilt, also nicht der Versicherungsnehmer den Ausschluss seiner Verantwortlichkeit, sondern der Versicherer darlegen und beweisen muss, dass er zum Zeitpunkt des Versicherungsfalls „schuldfähig“ gewesen ist [9]. Das soll daraus folgen, dass, anders als im Deliktsrecht, ohne grobes Verschulden das durch §61 VVG a.F./§81 VVG n. F. geschützte Interesse des Versicherers gar nicht berührt ist, der konkrete Haftungsgrund also schon nicht feststeht, wenn die Schuldfähigkeit des Versicherungsnehmers oder seiner Repräsentanten ungeklärt bleibt.

b) Die weit überwiegende Auffassung in Literatur und Rechtsprechung hält hingegen für richtig, §61 VVG a.F./\$81 VVG n.F. nur dann nicht anzunehmen, wenn der Versicherungsnehmer seine „Schuldunfähigkeit“ beweist [10]. ${ }^{23}$ Dem entspricht die Rechtsprechung des BGH. In seinem Urteil vom 22.02.198924 hat er die Annahme des Berufungsgerichts,

\footnotetext{
${ }^{21}$ Die Abkürzung CPAP steht für Continuous Positive Airway Pressure (Kontinuierlicher Atemwegsüberdruck)

22 BGHZ 39, 108; BGHZ 98, 139.

23 BGH RuS 1989, 349 = VersR 1989, 469/470 in Bestätigung von OLG Hamm VersR 1988, 394; OLG Hamm RuS 1988, 2; VersR 1988, 126; VersR 1992, 818; Rus 1998, 10; RuS 2001, 55; OLG Schleswig VersR 1994, 467.

24 IV a ZR 274/87, VersR 1989, 469, 470; ebenso Urt. v. 23.01.1985 - IV a ZR $128 / 83$ - r + s 1985, 80 = VersR 1985, 440
}

die Beweislastregel des $§ 827$ S. 1 BGB sei im Rahmen des $\S 61$ VVG entsprechend anzuwenden, für rechtsfehlerfrei erklärt. In seiner Entscheidung vom 20.06.1990 - IV ZR 298/89 - ${ }^{25}$ ist er gleichfalls davon ausgegangen. Für die zuletzt genannte Auffassung spricht, dass die Rechtsordnung, wie die gesetzgeberische Entscheidung des §827 S. 1 BGB zeigt, grundsätzlich von der Verantwortlichkeit einer Person ausgeht. Will jemand geltend machen, dass ein Ausnahmefall vorliegt, muss er dies darlegen und beweisen. Das gilt auch für das Verhältnis von Versicherungsnehmer und Versicherer. Dass der Versicherer grundsätzlich für durch einen Unfall verursachte Schäden an einem Kfz einstehen will und nur ausnahmsweise bei schwerem Verschulden das Risiko nicht zu tragen verspricht, steht der Annahme nicht entgegen, dass er aber von einer grundsätzlich bestehenden Verantwortlichkeit eines Versicherungsnehmers ausgeht.

Der BGH hat schon mehrfach ausgesprochen, dass die Beweislastregelung aus $§ 827$ S. 1 BGB, wonach den Täter die Beweislast für behauptete Unzurechnungsfähigkeit trifft ${ }^{26}$, auch im Rahmen von $§ 61$ VVG a.F./§81 VVG n.F. zu Lasten des Versicherungsnehmers Anwendung findet. ${ }^{27}$ Gelingt es dem Versicherungsnehmer nicht, den völligen Ausschluss der Verantwortlichkeit für den Zeitpunkt der Herbeiführung des Versicherungsfalls zu beweisen, so hat das Gericht im Weiteren davon auszugehen, dass eine solche Unzurechnungsfähigkeit nicht vorlag.

\section{Abmilderung des Vorwurfs grober Fahrlässigkeit bei verminderter Schuldfähigkeit \\ $\nabla$}

Hat der Versicherungsnehmer eine völlige Unzurechnungsfähigkeit nicht bewiesen, so kann der Tatrichter grundsätzlich aus dem Grad der objektiven Pflichtverletzung Rückschlüsse auf die innere Tatseite ziehen [11]. ${ }^{28}$ Er muss allerdings im Rahmen der gebotenen Gesamtwürdigung ${ }^{29}$ danach fragen, ob die Gründe, auf die der Versicherungsnehmer die (unbewiesene) Behauptung der völligen Unzurechnungsfähigkeit gestützt hat, Anhaltspunkte dafür geben, dass zumindest eine erhebliche Beeinträchtigung des Bewusstseins (unterhalb der Schwelle völliger Unzurechnungsfähigkeit) im Sinne einer erheblichen Verminderung der Einsichts- oder Hemmungsfähigkeit vorgelegen haben kann, die den Vorwurf in einem milderen Licht erscheinen lässt. In diesen Fällen kann grobe Fahrlässigkeit gegebenenfalls auf eine mittlere Form dieses Verschuldensgrades abgemildert werden $[3,12]$. Beruft sich ein Versicherungsnehmer beispielsweise auf eine Schlafstörung, die das Konzentrationsvermögen im Allgemeinen beeinträchtigt, so ist dies im Rahmen der Prüfung grober Fahrlässigkeit i.S. von §61 VVG a.F./§81 VVG n. F. gerade dann zu berücksichtigen, wenn nicht erwiesen ist, dass die Krankheit im Zeitpunkt der Herbeiführung des Versicherungsfalls zur völligen Unzurechnungsfähigkeit geführt hatte, weil daneben auch eine eingeschränkte Verantwortlichkeit des Versicherungsnehmers in Betracht kommen kann. ${ }^{30}$ Liegen solche Anhaltspunkte vor, trifft

\footnotetext{
25 BGHZ 111, 372

${ }^{26}$ BGHZ 98, 135 [136] = NJW 1987, 121; BGHZ 102, 227 [230] = NJW 1988, 822

27 BGHZ 111, 372 [374] = NJW 1990, 2387; BGH, NJW 1985, 2648 = VersR 1985, 440 und NJW 1989, 1612 = VersR 1989, 469.

${ }^{28}$ BGHZ 119, 147 [151 m. w. Nachw] = NJW 1992, 2418

${ }^{29}$ BGH, NJW 1989, 1612 = VersR 1989, 469; NJW 2003, 1118 = VersR 2003, 364 m. w. Nachw.

30 Vgl. dazu BGH, NJW 1989, 1187 = VersR 1989, 840.
} 
den Versicherer die volle Beweislast dafür, dass der Versicherungsfall dennoch grob fahrlässig herbeigeführt worden ist, die genannten, Zweifel begründenden Umstände also nicht wirksam geworden sind. ${ }^{31}$

\section{Fazit}

$\nabla$

Laut Angaben der Deutschen Gesellschaft für Schlafforschung und Schlafmedizin leiden mehr als 10 Prozent der deutschen Bevölkerung unter behandlungsbedürftigen Schlaf-Wach-Störungen. Das Krankheitsbild der obstruktiven Schlafapnoe wird wegen seiner insgesamt erschreckenden Häufigkeit in der Normalbevölkerung vor Gericht vermehrt als Ursache in Betracht gezogen werden.

Regelmäßig wird dem Schlafapnoiker, der nach einem - durch Einnicken verursachten - Verkehrsunfall den eigenen Fahrzeugschaden durch seine Kaskoversicherung erstattet verlangt, im Rahmen der Schadenregulierung vorgehalten, er habe den Verkehrsunfall grob fahrlässig verursacht. Einen allgemeinen Erfahrungssatz, dass einem „Sekundenschlaf“ immer Warnzeichen vorausgehen und aus diesem Grunde das gewöhnliche Maß an Fahrlässigkeit erheblich überschritten sei, gibt es jedoch nicht. Der Versicherer muss den Beweis führen, dass sich der Fahrer über die von ihm erkannten deutlichen Vorzeichen der Ermüdung bewusst hinweggesetzt hat. Das Missachten solcher sog. Prodormal-Erscheinungen indiziert grundsätzlich das Vorliegen grober Fahrlässigkeit. Die Einstandspflicht des Versicherers besteht hier nur noch - entsprechend der Schwere des Verschuldens des Versicherungsnehmers - prozentual gekürzt ( $\$ 81$ II VVG n.F.). Nach der höchstrichterlichen Rechtsprechung gehen im Falle des Einnickens infolge einer krankhaften (Schlaf-) Störung Ermüdungserscheinungen nicht zwangsläufig voraus. Allerdings muss der Betroffene seinen Krankheitszustand der Schlafapnoe beweisen. Ergibt sich im Rahmen einer Beweisaufnahme dagegen, dass er seine Schlafstörung kannte und sich nicht oder unzureichend behandeln ließ und so eine schwerwiegende Gefahr für den Straßenverkehr darstellte, so ist dem Versicherungsnehmer zum Vorwurf zu machen, nicht alle möglichen, geeigne- ten und zumutbaren Maßnahmen, wie etwa die Durchführung einer CPAP-Therapie, ergriffen zu haben. Dieses Unterlassen würde wiederum ein auch in subjektiver Hinsicht unentschuldbares Fehlverhalten und damit grobe Fahrlässigkeit darstellen.

Problematisch erscheint, ob der Versicherte bei ausgeprägter obstruktiver Schlafapnoe überhaupt schuldfähig war. Nach $§ 827$ S. 1 BGB ist schuldunfähig, wer einem anderen im Zustand der Bewusstlosigkeit einen Schaden zufügt. Ist der Betroffene in einen Sekundenschlaf ohne jede Vorankündigung gefallen, so trägt nach der Rechtsprechung des Bundesgerichtshofs der Versicherungsnehmer für die fehlende Verantwortlichkeit die Beweislast. Selbst wenn sich für eine völlige Unzurechnungsfähigkeit keine eindeutigen Beweise ergeben, so kann gleichwohl noch eine erheblich verminderte Einsichts- oder Hemmungsfähigkeit vorgelegen haben, die den Vorwurf einer groben Fahrlässigkeit abmildern kann.

\section{Literatur}

1 Terbille M (Hrsg) Münchener Anwaltshandbuch Versicherungsrecht, $\S 2$ Rn 365 - 367, 2. Aufl. München: 2008

2 Böhning W. Das Schlafapnoe-Syndrom - Ein wenig beachtetes Unfallrisiko - Schlafstörung und Tagesmüdigkeit als Unfallursache. NZV, 1997: $142 \mathrm{ff}$.

3 Knappmann U. Anwendbarkeit des $\S 61$ VVG bei Beeinträchtigung der Schuldfähigkeit durch Alkohol oder Drogen. NversZ, 1998: 13ff.

4 Römer W. Was bringt das neue VVG? r + s, 2008: 405ff.

5 Rixecker R. VVG 2008 - Eine Einführung. ZfS, 2007: 73

6 Marlow S, Spuhl U. Das neue VVG kompakt ein Handbuch für die Rechtspraxis. Verlag Versicherungswirtschaft, 2007: 72 - 74

7 Münstermann H.. Was der Anwalt in der Kasko- und Kfz-Haftpflichtversicherung wissen muss. Verkehrsrecht aktuell, 2008: $1 \mathrm{ff}$.

8 Fromm I. Die Neuerungen in der Fahrerlaubnisverordnung (FeV) zur Bekämpfung der Gefährdung des Straßenverkehrs durch Schlafapnoe und sonstige chronische Schlafstörungen. Pneumologie 2008; 62: $387 f f$

9 Prölss J, Martin A. Versicherungsvertragsgesetz, Kommentar, §61 Rdnr. 25, 27. Aufl. München: 2004

10 Römer W, Langheid T. Versicherungsvertragsgesetz, Kommentar, §61 Rdnr. 81, 2. Aufl. München: 2003

11 Römer W. Das sogenannte Augenblicksversagen. VersR, 1992: 1187ff.

12 Lang A. Alkohol im Straßenverkehr und Versicherungsschutz. NZV, 1990: $169 \mathrm{ff}$. 